\title{
LEAN DESIGN IN HYDRAULIC INFRASTRUCTURE - RIVER DEFENSES AND DIKES - A CASE STUDY FROM PERU
}

\author{
Frank Chuquín', Cristhian Chuquín², and Romina Saire ${ }^{3}$
}

\begin{abstract}
The construction sector has been changed in different aspects since the implementation of best practices of lean construction and others. It is crucial to remark that those new methodologies have been trying to address construction issues related to the execution part but with little attention to the design stage.

In Peru, the use of lean construction started as part of an initiative from the private sector and specifically in the execution part. In that sense, lean design was introduced later and always by the private sector. Little by little the public sector started to get used to lean construction. Nevertheless, in hydraulic infrastructure such as river defenses and dikes the progress of introduction lean design has been insignificant in the country. This paper describes step by step the implementation of lean design in capital projects related to hydraulic infrastructure in Peru specifically for river defenses and dikes.It is the objective of this paper to address the difficulties founded in the implementation and what strategies have been deployed in order to overcome those barriers. Two tools of lean design that were used are: set based design and value stream mapping along with concepts of change management.
\end{abstract}

\section{KEYWORDS}

Change management, lean design, set based design, value stream,. hydraulic infrastructure.

\section{INTRODUCTION}

The construction of public infrastructure in Peru is characterized by a lack of certainty about when the project is going to finish and at what the total budget will be at the end of the execution part, this is also closely related to corruption. As a result, society suffers from not having the necessary infrastructure such as hospitals, roads, schools, bridges, and hydraulic infrastructure. In 2017 the coast of Peru suffered intense damage due to heavy rains and enormous areas were flooded, the total damage was estimated at 384 millions dollars only in hydraulic infrastructure. A governmental institution was created

Lecturer, Researcher at Construction Management \& Technology Research Group (GETEC), Pontifical Catholic University of Peru, Lima, Peru, frank.chuquin@pucp.pe, orcid.org/0000-00018342-6602

2 Civil Engineer, Researcher at Construction Management \& Technology Research Group (GETEC), Pontifical Catholic University of Peru, Lima, Peru, chuquin.cr@ pucp.edu.pe, orcid.org/0000-0002$\underline{4080-9600}$

3 Research Assistant at Construction Management \& Technology Research Group (GETEC), Pontifical Catholic University of Peru, Lima, Peru, rsairecanales@gmail.com, orcid.org/0000-0002-2433-1301 
in order to be responsible for protecting people from floods. Then, in 2020 the deployment of resources in order to start the design and construction of river defenses and dikes started in a fast track manner.

According to Forbes and Ahmed (2011) poor design and documentation quality have been identified as a major factor in reducing the overall performance and efficiency of construction projects. Moreover, Hill et al. (2017) report that the design stage can have more opportunities to reach better engineering solutions.Then, design can have a bigger impact in the cost and time of a project as well as the opportunity of the changes which are aligned with the MacLeamy curve. It was important to include methodologies or best practices that can ensure that those objectives (cost and time) will be reached.

Ballard and Howell (2003) pointed out that lean project delivery system (LPDS) provides a means of improving the entire design and construction process. As it is known, LPDS consists of five phases: project definition, lean design, lean supply, lean assembly and use. The lean design phase comprises three processes: design concept, process design and product design. As it is stated by Seed (2018) lean/Integrated Project Delivery is a response to the dissatisfaction of what we have in the construction industry. Therefore, the lean project delivery system was chosen as a framework for developing the lean design management. In particular two tools were included as part of the design stage: value stream mapping and set based design. Moreover, Mota et al. (2019) pointed out that there are limitations in major infrastructure projects when implementing lean design management such as: lack of collaboration, insufficient knowledge and rigidity of the organization.

Firstly, Kanai and Fontanini (2020) mentioned three positive factors of the value stream mapping: it helps to visualize the whole process, it is a manner to identify waste. The map is a first step for an implementation. Then, the description of the entire design process is not included in traditional design offices. Even, the most experienced organizations in Peru do not use a value stream mapping. Therefore, this tool was introduced to the technical team in order to generate this flow process with the input of each discipline. This tool is aligned with the process design part of lean design.

Secondly, Sacks et al. (2010) explain it is essential to invest time in the concept design phase and not to rush into the design detail. Besides, Forbes and Ahmed (2011) reported that a technique suggested for lean design is to pursue a set based design strategy. Then, the optimal solution is the result of different alternatives of design and the fact that creating different scenarios and the analysis of them can take us to a better solution. Therefore, this search of the optimal solution is included through the concept of set based design which played a pivotal role in finding this best alternative. This tool is aligned with the product design part of lean design.

Apart from those tools mentioned in the paragraphs above and its importance as a medium to implement lean design from the technical point of view. This case study explores the managerial aspect of this endeavor. In that regard, Wandahl (2014) pointed out that although several companies are introducing new management concepts, most of them fail in implementing them due to their internal culture. Therefore, this paper explains the barriers found in the implementation of lean design from the perspective of change management. It was notorious the natural resistance from people who were used to work in a traditional framework which means without tools from lean design.

In this regard, Kotter (1997) explains how to sustain in the long term any change in a company through the implementation of the methodology named the eight steps of Kotter. Moreover, Fischer et al. (2017) state it is necessary to repeat the strategic steps 
periodically, because executive managers need to remember lessons learned. In that context, the authors recognized that in order to reach the implementation inside an organization it is necessary to address a change management strategy.

\section{KEY CONCEPTS}

\section{Change Management}

As part of social studies this concept gives us insights about how to defeat barriers when we try to apply new ideas, concepts and methodologies inside an organization. According to Zimmermann (2000) any important change will have resistances or difficulties to overcome. It is crucial to keep in mind that it is necessary to include leadership in all the processes of change. This model of implementation or transformation requires sacrifice, dedication and creativity. None of those elements are close friends with coercion. It will be important to keep in mind those eight steps mentioned by John Kotter in his book "Leading change" (1997) in order to really create an environment in which change is possible, and it will keep going in the long run. Those steps are the following: create a sense of urgency, create a guiding coalition, create a vision, communicate vision, remove barriers, generate quick wins, sustain the pace and make it stick. This is aligned to Schein (2009) who pointed out that change leadership must comprise: credibility, clarity of vision, ability to articulate the vision, understanding of cultural dynamics and process skills.

\section{LEAN ProjeCt DeliVery SySTEM (LPDS)}

It comprises five phases that juxtaposes them and those phases influence each other which creates a necessary integration between different stakeholders (Forbes and Ahmed, 2011). The lean design phase comprises: conceptual design, process design and product design.

\section{SET BASED DESIGN (SBD)}

It is a design method in which sets of alternative solutions are evaluated until the last responsible moment (Hill et al., 2017). This last responsible moment should not impact in a negative manner the duration of the design process. Ballard (2000) precises the definition of this last responsible moment as the "point at which failing to make the decision eliminates an alternative". Moreover, Parrish et al. (2007) consider this tool improves the stakeholders engagement in order to increase the value generated by eliminating rework.

\section{VALUE STREAM MAPPING (VSM)}

As stated by Orihuela et al. (2015) VSM is a visual tool which permits teams to correctly identify how the flow processes are executing. Additionally, Kanai and Fontanini (2020) pointed out VSM helps to comprehend the flow of material and information. It is a tool that permits to know step by step the entire process of a work. It permits mapping the flow of value. Through this tool anyone in a team (i.e. a designer) can understand when and how his contribution helps to reach the goal of having the design done. By knowing how each process interacts with others and translating this idea on how different specialists, designers or stakeholders are able to have a holistic point of view of the design process and even they could make suggestions of improving the entire process through innovation and creativity. This tool permits global optimization and not only local ones because it is easier to notice the presence of waste in the whole process. This is supported by Orihuela et al (2015) by stating that VSM will reduce waste at the design phase by 
preventing negative iterations and rework.In that sense, the use of a value stream mapping leads to a continuous improvement.

\section{HYPOTHESIS}

The implementation of a structured strategy of change management in a governmental institution in Peru (that is in charge of the design and construction of hydraulic infrastructure) will permit to develop new methodologies such as lean design management and tools such as value stream mapping and set based design as manner to reach effectiveness and efficiency in the delivery of hydraulic infrastructure projects such as river defenses and dikes.

For this case study, the research questions are the following: how feasible is the implementation of lean design in hydraulic public projects? and how to overcome the technical and managerial barriers?

\section{METHODOLOGY OF IMPLEMENTATION}

There were different meetings with the directives of the governmental institution, designers and project managers and specialists in order to explain the necessity to incorporate a lean design methodology, as a manner to assure that the engineering solution reached was the most suitable and optimal, considering a multi-criteria analysis and a cost-benefit analysis. In order to reach the necessary support for this initiative we followed the eight steps (figure 1) described in Kotter (2012) including the following considerations:

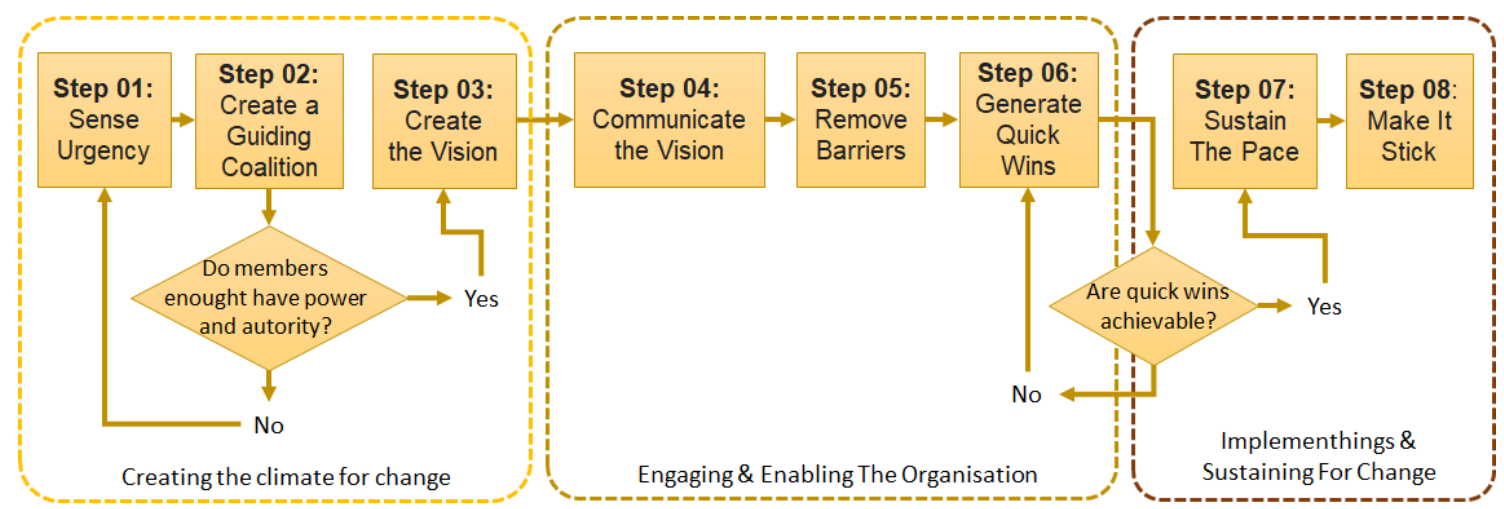

Figure 1: Steps of the methodology (adapted from Kotter 2012)

First, sense of urgency: In 2017, the northern part of Peru suffered flood damage and still in 2020 there was no definitive infrastructure designed to protect people and agricultural areas. Then, to convey the sense of urgency, senior management was sensitized by watching videos of the damage that occurred and the impact on the population. This message immediately reached directors, executives, project managers, designers and specialists who work in this institution and who are responsible for the design and construction of protective hydraulic infrastructure such as river defenses and dikes.

Second, create a guiding coalition: The power of position is crucial for the success of the endeavor of using new methodologies as lean design, set based design and value stream mapping. In this sense, it is essential to get the support of the organization's executives. Moreover, to strengthen this coalition, workshops must be offered to the members of the coalition to be trained in the use of lean design management. 
Parallel that, not only bringing in people who have some experience in similar systems and have worked in academia enhances this guiding coalition, but also part of this coalition is the academy and it is important to prioritize an alliance between academia and the industry (institution).

Third, create a vision :This has to show a clear direction to all stakeholders because it increases motivation and makes changes in the design process much easier. Then, keeping in mind the urgency of protecting people from the effects of natural disasters, some informal meetings and two formal meetings were held with coalition members to create the vision. Consequently, this resulted in a clear, communicable and achievable vision that emphasizing how essential it was to quickly complete the design and construction of the hydraulic infrastructure to high standards with all talented individuals considered.

Fourth, to communicate vision: it is crucial that not only the coalition team knows the vision, but also most of the people involved in the change. Then, in each of the weekly meetings with those involved, structured speech emphasized the importance of combining the use of a lean approach to design. Workshops were given to explain the tools (SBD and VSM). Technical guides were also written in order to explain more about the tools to be used.

Fifth, remove barriers: Management change includes barriers that must be removed. For this, it is necessary to show the need of urgency to the actors to sensitize them and commit them to the cause. Likewise, optimal solutions must be offered. In this case study, the problem was the deficiencies generated by using the traditional work methodology. That is why it was necessary to explain and demonstrate the benefits of using the new methodology to gain more allies in the process.

Sixth, generate quick wins: in order to generate trust in the environment and help to consolidate the process, it is necessary to plan quick wins. In this particular case study the first quick win was to gain the support of the organization's executives. A second win was when we had onboarding members to the coalition team such as the design manager and construction manager. Then, the first draft of the value stream mapping was a quick win because it demonstrated how helpful and important it was to have the opportunity to see in detail the entire process. The next win was when some specialists such as the archaeologist and the landscaping architect started spreading the work with other stakeholders. These quick wins demonstrated the positive effects of the new methodology and not only increased the momentum of the people involved in the change, but also they were recognized by people who were against the changes and consequently, they changed their attitude.

Seventh, sustain the pace: Once those quick wins were reached the coalition team was more sure that people were compromised with a further analysis of flow processes by categorizing in three different components: customer value-added, business valueadded and non value-added. Also, in order to sustain the pace of the implementation, designers and specialists created a more collaborative environment in which the goal was to deliver a leaner design process. At this point, the coalition team found more advocates between the participants which facilitated the implementation process.

Eighth, make it stick: The ultimate goal of an implementation is to change the culture in terms of norms of behavior and shared values of the organization. This involves a longterm commitment. So, we need to assure quick or early wins in order to inspire people to continue with their effort. In this sense, communication is critical because people need to be reinforced about the objectives of the changes. In this case study the long-term 
commitment will be visible when this new manner to work in the design process will be used in each project in charge of the hydraulic infrastructure.

\section{RESULTS}

As a result of the implementation, the strategy focuses on change management and the collaboration of the coalition team and advocates. In the following, it summarizes different aspects of the implementation processes such as: strategies for implementing lean design management (SBD and VSM), facilitating elements for implementation, difficulties and barriers found and strategies for overcoming difficulties. Then, each step of the framework is evaluated from those four dimensions.

\section{SENSE OF URGENCY}

Strategies for implementing lean design management: People were told about the delay in these projects. The flood damages occurred in 2017 and still in 2020 we have not had a final solution for protecting people and infrastructure in urban cities and agricultural areas.

Facilitating elements for implementation:Directives, executives, project managers, designers and specialists were aware and conscious about the necessity of these projects.

Difficulties and barriers found: It was difficult to transform this sense of urgency in activities and measurable tasks that permits a call to action.

Strategies for overcoming difficulties: In formal meetings a topic of discussion was to define milestones of the design and the time frame.

\section{Create A Guiding Coalition}

Strategies for implementing lean design management: To get people involved with authority and power inside the organization.

Facilitating elements for implementation: Two of the executives: the construction manager and the design manager on board of this implementation helped to propel it.

Difficulties and barriers found: To convince the executive director, construction manager and design manager that it is worth implementing

Strategies for overcoming difficulties: To explain best international practice of implementing new methodologies such as lean design management.

\section{Create A Vision}

Strategies for implementing lean design management:To create a message about the importance of finishing as soon as possible and at the same time with high standards the design and construction of hydraulic infrastructure that can protect people from death and poverty.

Facilitating elements for implementation: The vision created was communicable, clear and most importantly feasible considering all the talented people involved in this endeavor.

Difficulties and barriers found: Multidisciplinary projects like this one need to address many concerns coming from each discipline. There were eleven disciplines.

Strategies for overcoming difficulties: Through one to one meetings with each specialist we sought to understand the concerns and to figure out how to include them in the vision. 


\section{To Communicate The Vision}

Strategies for implementing lean design management: There were three key elements that helped to communicate the vision: multiple forums, repetition and bilateral communication. As part of the strategy there were seven workshops delivered to stakeholders (executives, directors, project managers, designers, specialists) in which the methodology of lean design. in particular value stream mapping and set based design were explained.

Facilitating elements for implementation: In informal meetings the coalition team delivered the message, the repetition of the message was important. Also, it was convenient to be open to feedback and to stimulate this bilateral communication between people involved and the coalition team. Also, the vision was repeated at the beginning of each weekly meeting.

Difficulties and barriers found: The problem was to recognize at what level people were understanding the vision (message).

Strategies for overcoming difficulties: Surveys were implemented in order to measure at what extent people were engaged to the vision.

\section{REMOVE BARRIERS}

Strategies for implementing lean design management: It was a priority to identify barriers, so through surveys we collected data about the level of knowledge and the flexibility of implementing new tools by the stakeholders. Then, a schedule of training was developed. The selected topics were change resistance and technical tools such as set based design and value stream mapping.

Facilitating elements for implementation: The support of the guiding coalition was fundamental for implementing the strategy in this step. Also, the specialized knowledge of the consultants from academia was important.

Difficulties and barriers found: There were two types of barriers such as: structural impediments and skills-knowledge obstacles. Those structural barriers were related to the traditional design process in which there is no room for evaluation of different design alternatives and it is not appreciated to develop and share the mapping of the value creation. Also, not knowing what lean design is about or its tools is the second type of barrier categorized as insufficient skill and knowledge.

Strategies for overcoming difficulties: Through extensive workshops, technical notes and a clear communication, the knowledge gap was fullfill and the structural barrier was overcome.

\section{Generate Quick Wins}

Strategies for implementing lean design management: To set clear goals which comply with the following characteristics: measurable, achievable and quantifiable. Therefore, planning how to achieve those goals was important and to get into details such as: resources, time and constraints. It was established goals of first level and goals of second level of detail. Those of the first level were each step of Kotter framework. Second level goals were related to the internal process with all the stakeholders.

Facilitating elements for implementation: The framework designed by Kotter gave a clear path of how to establish those goals. Those eight steps were taken initially as measurable goals.

Difficulties and barriers found: The elaboration itself of the value stream mapping was difficult because of the number of participants. Each specialist from his/her point of 
view weighted differently the time, importance and precedence of each activity. Also, meetings with all people involved were relevant, but initially those meetings took more working hours than expected.

Strategies for overcoming difficulties: One to one meetings were established. Those helped to understand the point of view of each specialist. From this, a first draft of the value stream was proposed. This was a manner to decrease the duration of meetings with all people involved.

\section{Sustain The PaCe}

Strategies for implementing lean design management: Weekly meetings were established between members of the guide coalition and specialists. In each meeting a general perspective was given about where we are in the roadmap (quick wins). Then, a gap analysis between actual progress and planned progress. Also, discussion about constraints and an analysis of foreseen activities.

Facilitating elements for implementation: Those initial advocates helped to increase confidence in other participants.

Difficulties and barriers found: Attendance at those weekly meetings was a big issue at the beginning of implementation.

Strategies for overcoming difficulties: To those people who were absent at that weekly meeting, it was necessary to schedule a one to one meeting in order to increase awareness and commitment.

\section{MAKe IT STICK}

Strategies for implementing lean design management: The strategy was to implement this new methodology in each project in charge of the institution. The portfolio consists of nine projects such as: Cañete river, Huaura river, Matagente river, La Leche river, Motupe river, Tumbes river, Casma river, Huarmey river and Mala river. Therefore, through repetition we expected to improve the design management and make it repeatable in each project.

Facilitating elements for implementation: By having a portfolio of nine projects , this gives us the opportunity to use lessons learned from previous projects. This is because each project has a differentiated start.

Difficulties and barriers found: Since each project had its own personnel who did not make the effort of implementation from the beginning this makes it difficult at the outset. Also, people from each region were far away from the central office. Then, virtual meeting were commonly used.

Strategies for overcoming difficulties: Training and workshops were given for people in each region. Apart from virtual meetings, a physical one was established once a month.

\section{DISCUSSION}

The feasibility of implementation is analyzed in two levels at the global and local one. At the global level the most important barriers were: the decision at the executive level of implementing a lean design management approach, the conformation of the coalition team, the planning of the workshop-training to specialists and to plan which those quick wins will be. Nevertheless, the governmental institution took more time than expected just to consider the implementation or not of lean design management. It was expected that in 2 weeks the decision would be made, but it took 6 weeks just to start. The 
importance of having an alliance between the governmental institution with academia in this endeavor played a pivotal role. This is because the guidance from scholars helped the path of the coalition team.

At the local level, two elements were closely related: communications and the restrictions for pandemic in the country. This virtualizes the work because the restriction of having a limited number of professionals at the office propelled the use of virtual teams. The use of technology eases the implementation because people could join training sessions and workshops from their home. Also, the level of commitment increased when people started seeing the results of applying lean design. At the end of the design process of one of the projects, a survey was applied and it was found that $72 \%$ believed the quality of their work increased and the rework decreased.

There were eleven different types of specialists for each project: topography, geology, archeology, environmental, geomorphology, geotechnics, hydrology, hydraulic, social, landscaping and civil works. What those specialists expressed in the survey at the end of the design process was the importance of knowing when to start interacting with other specialists and what they expected from the previous process is what they highlighted as valuable in the implementation of lean design.

Considering there is a portfolio of nine projects, this gives the opportunity to learn from repetition and from the lessons learned of previous projects.

\section{CONCLUSIONS}

Those difficulties that we encountered were mainly related to the structure of the organization. It was a governmental institution which was in charge of the design and construction of hydraulic infrastructure. Then, the feasibility of the implementation had an important barrier to overcome which is related to how executives and directors used to work in a traditional framework and it took a long time to make a decision to implement it. Also, the organizational structure did not allow to allocate new staff with experience in lean design. Then, the alliance with academia was important for a successful implementation.

Despite those challenges, directors, executives and specialists were convinced that knowing the entire process using a tool named value stream mapping and being aware of each interaction between different disciplines will bring more chances to obtain an optimal engineering solution from a cost benefit analysis that guarantees a better value for money.

The use of a structured framework such as the eight steps of change management helped to overcome the technical and managerial barriers. It was found that each of the steps are important in order to protect the implementation from detractors and create the environment for those who advocate for this new methodology of lean design. The framework was used as a guide of each step in the entire process of implementation. Two of the most relevant steps that were highlighted by stakeholders in a survey were the coalition team and the establishment of quick wins. Both were crucial for the implementation and for keeping the interest of all designers, specialists, executives, directors, project managers and design managers.

It will be important for future research to analyze at the end of the construction stage how the decision made in the design influences the result of the execution part. 


\section{ACKNOWLEDGMENTS}

We would like to thank in advance all participants in this case study from designers, specialists, directives, executives, project managers and design managers. We would also like to thank the openness of the governmental institution and its desire to achieve excellence.

\section{REFERENCES}

Ballard, G. (2000). "Positive vs Negative Iteration in Design". Proc. 8th Ann. Conf. of the Int'1 Group for Lean Construction (IGLC-8), Univ. of Sussex, Brighton. UK. 4455.

Ballard, G. and Howell, G. (2003). Lean Project Management. Building Research \& Information. 31(2) 119-133.

Fischer M., Ashcraft H., Reed, D. and Khanzode, A.(2017). "Integrating project delivery", John Willey and Sonc Inc, New Jersey, USA.

Forbes, L.H. and Ahmed, S.M. (2011). "Modern Construction: lean project delivery and integrated practices", Taylor and Francis Group Inc., Florida, USA.

Hill, K., Copeland, K., Pikel, C. (2017). "Target Value Delivering: Practioner guidebook to implementation", Lean Construction Institute, Virginia, USA.

Kanai, J. and Fontanini, P.S.P. 2020. "Value Stream Map and Visilean for Prefabricated Concrete Panels

Kotter J. (1997). "El lider del cambio", McGraw-Hill Interamericana Editores. México.

Management." Proc. 28th Annual Conference of the International Group for Lean Construction (IGLC). Berkeley, California, USA, pp 673-674".

Mota, B., Biotto, C., Choudhury, A., Abley, S. and Kagioglou, M. (2019). "Lean Design Management in a Major Infrastructure Project in UK". Proc. 27th Annual Conference of the International Group for Lean Construction (IGLC). Dublin, Ireland, 3-5 Jul 2019. pp 37-48.

Orihuela, P., Orihuela, J. and Pacheco, S. (2015). "Communication protocol for implementation of Target Value Design (TVD) in building projects". Procedia Engineering, Creative Construction Conference 123, 361-369.

Parrish K., Wong, J., Tommelein, I., and Stojadinovic, B. (2007). "Exploration of SetBased Design for Reinforced Concrete Structures", In Proc. 15th Ann. Conf. of the Int'1 Group for Lean Construction (IGLC-15), Michigan, USA. 213-222.

Sacks R.; Koskela L.; Dave B.; and Owen R. (2010) "Interaction of Lean and Building Information Modeling in Construction" Journal of Construction Engineering and Management, Vol. 136, No. 9, September 1, 2010.

Schein, E. (2009). "The corporate culture survival guide". John Wiley \& Sons, San Francisco, USA.

Seed, W.B. et al (2018). "Transforming Design and Construction: A Framework for Change", Lean Construction Institute, Virginia, USA.

Wandhal, S. (2014). "Lean Construction with or without Lean-Challenges of implementing" Lean Construction. In Proceedings of the 22nd Annual Conference of the International Group for Lean Construction, Oslo, Norway, 97-108.

Zimmermann (2000) “Gestión del Cambio Organizacional”. Ediciones ABYA-YALA, Quito, Ecuador. 